\title{
Association Between Vedolizumab Levels, Anti-vedolizumab Antibodies, and Endoscopic Healing Index in a Large Population of Patients with Inflammatory Bowel Diseases
}

\author{
Andres J. Yarur ${ }^{1}$ (1) $\cdot$ Parakkal Deepak $^{2} \cdot$ Niels Vande Casteele $^{3} \cdot$ Robert Battat $^{4} \cdot$ Anjali Jain $^{5} \cdot$ Lauren Okada $^{5}$. \\ Mark Osterman $^{6} \cdot$ Miguel Regueiro $^{7}$
}

Received: 12 September 2020 / Accepted: 8 October 2020 / Published online: 22 October 2020

(c) The Author(s) 2020, corrected publication 2020

\begin{abstract}
Background The aim of this study was to assess the relationship between serum vedolizumab (VDZ) concentrations and antibodies to VDZ (ATV) in a large cohort of patients with inflammatory bowel diseases. Furthermore, we evaluated the association between serum VDZ concentrations and a novel serum-based biomarker panel designated as the endoscopic healing index (EHI), developed and validated for identifying mucosal inflammation in patients with Crohn's disease (CD). Methods Retrospective study where results from patient samples submitted to a commercial clinical laboratory were included. Serum VDZ and ATV levels were analyzed using a drug-tolerant assay. In CD patients for whom both VDZ and EHI were available, VDZ concentrations were correlated with EHI. serum VDZ threshold analysis was performed using ROC curves, and the serum VDZ concentrations that best differentiated EHI $<20$ (previously associated with endoscopic remission) were chosen.

Results A total of 9356 patients were included in the VDZ/ATV analysis. Detectable ATV was observed in 2.9\% patients with significantly lower serum VDZ concentrations when compared to those with undetectable ATV [3.9 $\mu \mathrm{g} / \mathrm{mL}(0-9.0)$ vs. $11.3 \mu \mathrm{g} / \mathrm{mL}(5.9-20.6), p<0.0001]$. Of the patients with serum VDZ result, 287 patients had a concomitant EHI test. An inverse correlation was observed between VDZ concentration and EHI (rho $=-0.20, p<0.001)$. A serum VDZ concentration $\geq 15.7 \mu \mathrm{g} / \mathrm{ml}$ was best correlated wi th an EHI <20 [AUROC: 0.67 (95\% CI 0.57-0.77)].

Conclusions Incidence of ATVs was low, but significantly associated with lower VDZ levels. A serum VDZ concentration threshold of $\geq 15.7 \mu \mathrm{g} / \mathrm{ml}$ was associated with endoscopic remission as defined by an $\mathrm{EHI}<20$.
\end{abstract}

\footnotetext{
Update 11 November 2020 In the original article, the third author name, 'Niels Vande Casteele' was published with 'Niels Vande' as the first name and 'Casteele' as the surname. The corrected first name and the surname of the author is Niels Vande Casteele Niels (first name); Vande Casteele (surname).
}

Electronic supplementary material The online version of this article (https://doi.org/10.1007/s10620-020-06669-6) contains supplementary material, which is available to authorized users.

Andres J. Yarur

ayarur@mcw.edu

1 Division of Gastroenterology and Hepatology, Medical College of Wisconsin, 701 Watertown Plank Road, Milwaukee, WI 53226, USA

2 Division of Gastroenterology and Inflammatory Bowel Diseases Center, Washington University in Saint Louis School of Medicine, St. Louis, MO, USA

3 University of California, San Diego, La Jolla, CA, USA
4 Division of Gastroenterology and Hepatology, Weill Cornell Medicine, New York, NY, USA

5 Prometheus Biosciences, San Diego, USA

6 Division of Gastroenterology and Hepatology, University of Pennsylvania Perelman School of Medicine, Philadelphia, PA, USA

7 Division of Gastroenterology and Hepatology, Cleveland Clinic, Cleveland, OH, USA 
Keywords Vedolizumab levels · Anti-vedolizumab antibodies $\cdot$ Crohn's disease $\cdot$ Ulcerative colitis $\cdot$ Inflammatory bowel diseases $\cdot$ Endoscopic healing index

\section{Background}

Vedolizumab (VDZ) is a humanized immunoglobulin G1 monoclonal antibody to $\alpha 4 \beta 7$ integrin that modulates intestinal, but not brain, lymphocyte trafficking. The drug is more effective than placebo in the induction and maintenance of remission in patients with ulcerative colitis (UC) and Crohn's disease (CD) $[1,2]$ As with other biologics for inflammatory bowel diseases (IBD), patients can lose response after experiencing a benefit (secondary non-response), while others never respond (primary non-response).

Previous experience with anti-tumor necrosis factor (TNF) drugs has shown that primary and secondary nonresponse may be associated with low drug levels and/or the presence of anti-drug antibodies [3-5]. As new monoclonal antibodies become available, therapeutic drug monitoring (TDM) may yield insight into loss of response and biologic optimization. Observational studies exploring associations between VDZ pharmacokinetics and efficacy have been conflicting. Most have shown that early (week 6) VDZ levels correlate with clinical and endoscopic outcomes [6-8]. All studies have demonstrated a low prevalence of anti-VDZ antibodies (ATV), especially when compared to anti-tumor necrosis factor (TNF) agents [6, 8]. The low VDZ immunogenicity rates and small sample sizes have precluded meaningful exploration of ATV on VDZ pharmacokinetics and efficacy.

Treat-to-target has emerged as an important treatment strategy in IBD patients. The primary outcome of this approach is objective improvement of gastrointestinal inflammation that is not reliant on the subjective nature of symptom-based treatment alone [9]. The gold standard target for inflammation improvement has been endoscopic mucosal healing, which has also been associated with better long-term outcomes [10]. Endoscopic evaluation is invasive and not practical to repeat frequently in a short period of time, limiting its use. Hence, there is a need for non-invasive biomarkers of intestinal inflammation. Recently, a 13-biomarker panel assay in serum was developed and validated as an adjunct biomarker of endoscopic disease activity in patients with CD [11]. Endoscopic healing index (EHI) demonstrated better sensitivity/specificity than serum C-reactive protein (CRP) and equivalent sensitivity/specificity to fecal calprotectin (FC) (Monitr ${ }^{\mathrm{TM}}$; Prometheus Biosciences, San Diego, CA, USA) [11]. Even though previous studies have shown an inverse correlation between biologic drug levels and both CRP and FC, the association between VDZ levels and the EHI is unknown.

The current study had two specific aims. The first was to assess the prevalence of ATV in a large real-world IBD population and investigate the association with serum
VDZ drug levels. The second was to perform an exposure-response analysis correlating VDZ drug levels and ATV with the EHI test in CD patients.

\section{Materials and Methods}

\section{Study Design and Setting}

This was a retrospective study using data from a commercial clinical laboratory (Prometheus Biosciences) accredited by the American College of Pathologists. Patients were included if a VDZ/ATV test was performed as part of the commercial laboratory testing and if they ever reported a diagnosis of inflammatory bowel disease as per ICD codes (Table 1). In the sub-group analysis comparing UC and CD patients, those patients with discordant diagnostic codes were not used. For the second part of the analysis on EHI and VDZ/ATV, a subgroup of patients was identified that excluded a diagnosis of UC and had a concomitant EHI measurement.

\section{Evaluations and Input Data}

Available patient data included age, gender, and the ICD code-based diagnosis $(\mathrm{CD}, \mathrm{UC}$, indeterminate colitis, or pouchitis). All samples had serum measurements of VDZ and ATV levels. The sub-cohort that had a concomitant EHI measurement was analyzed separately [11].

\section{Measurement of Vedolizumab Levels and Anti-vedolizumab Antibodies}

All patient samples were analyzed for standard of clinical care. VDZ concentrations were measured using a validated drug-tolerant assay specific for VDZ that utilized the previously described homogeneous mobility shift assay (HMSA) methodology [12]. The lower limits of quantitation for VDZ and ATV were $1.6 \mu \mathrm{g} / \mathrm{mL}$ and $1.6 \mathrm{U} / \mathrm{mL}$, respectively, while the upper limits were $40 \mu \mathrm{g} / \mathrm{mL}$ and $75 \mathrm{U} / \mathrm{mL}$, respectively.

VDZ concentrations of $15 \mu \mathrm{g} / \mathrm{mL}$ were found to have the best sensitivity/specificity in previous studies using HMSA, demonstrating this threshold to be associated with clinical remission [13].

\section{Endoscopic Healing Index}

Patients who had concomitant VDZ levels and EHI values within 30 days of each other and excluded a diagnosis of UC were included in a sub-analysis VDZ-EHI cohort. The test was developed as previously described [11]. 
Table 1 Baseline characteristics of the study population

\begin{tabular}{lll}
\hline & Anser $^{\circledR}$ VDZ & $\begin{array}{l}\text { Monitr }{ }^{\mathrm{TM}}+\text { Anser } \\
\text { VDZ (sub-cohort) }\end{array}$ \\
\hline Number of patients & 9356 & 287 \\
Number of Anser $^{\circledR}$ VDZ samples & 12,595 & 287 \\
Female gender (\%) & $5205(55.6)$ & $168(58.5)$ \\
Diagnosis of Crohn's disease (ICD codes: 555., K50.) (\%) & $5059(54.1)^{\mathrm{a}}$ & $282(98.3)$ \\
Diagnosis of ulcerative colitis (ICD codes: 556., K51.] (\%) & $4648(49.7)^{\mathrm{b}}$ & $0(0)$ \\
Diagnosis of indeterminate colitis (ICD codes: 558.9, K52.3) (\%) & $135(1.4)^{\mathrm{c}}$ & $0(0)$ \\
Diagnosis of pouchitis (ICD codes: 569.7, K91.85) (\%) & $47(0.5)^{\mathrm{d}}$ & $0(0)$ \\
Age, entire cohort (median in years) (IQR) & $42(28-58)$ & $48(36-62)$ \\
Patients < 18 years (\%) & $614(6.6)$ & $11(3.8)$ \\
Median age, patients $<18$ years (median in years) (IQR) & $15(12-16)$ & $16(13-17)$ \\
\hline
\end{tabular}

${ }^{a} 4,614 / 5059$ (91.2\%) patients had only CD ICD codes while the remaining 445 patients had overlapping ICD codes for UC, IC, and/or pouchitis

${ }^{\mathrm{b}}$ Of the 4648 patients with UC ICD codes, 4190 patients (90.1\%) only had UC codes

${ }^{c}$ Only 36/135 (26.7\%)

${ }^{\mathrm{d}} 11 / 47$ (23.4\%) patients were unique for IC or pouchitis codes, respectively; overall, 8851/9356 (94.6\%) patients had a unique ICD code
The EHI scores range from 0 to 100 units, where higher scores denote a higher burden of CD endoscopic activity. The test was validated in two independent CD cohorts with high diagnostic accuracy. A serum EHI cutoff value of $<20$ points identified patients in endoscopic remission with a high level of sensitivity in various patient populations (83.2-97.1\%), and specificities of 36.6-69.0\%, respectively. A cutoff value of $>50$ points identified patients with endoscopic active disease with a high specificity (87.8-100\%), with sensitivities of 30-37\%. Because of the high sensitivity, in this study, we considered endoscopic remission as an EHI $<20$, while an EHI $>50$ was considered as endoscopic active disease.

\section{Statistical Analysis}

Descriptive statistics were used to examine the baseline characteristics of the study population. Continuous variables were compared using the Mann-Whitney $U$ test or Kruskal-Wallis test with post hoc Dunn's test (for nonparametric variables). Patient level analysis used the first available sample or the first available ATV-positive sample for the analysis. The $\chi^{2}$ test was used to evaluate distributions of categorical variables. Correlation between serum VDZ concentrations and other continuous variables were performed using Spearman's rank correlation test. Receiver operating characteristic (ROC) curves were evaluated to identify VDZ concentration thresholds with the best sensitivity/specificity (Youden's index) associated with an $\mathrm{EHI}<20$. A $p$ value $<0.05$ was considered statistically significant. Analysis was carried out using R v.3.6.2.

\section{Results}

\section{Patient Characteristics}

Within the CLIA dataset, 9356 unique patients were identified who provided serum samples for VDZ assay measurement between October 2016 (test launch date) and August 31,2019 , and were included in the analysis. The median age was 42 years (IQR: 28-58), 5205 (55.6\%) were women, and $6.6 \%$ were pediatric patients (less than 18 years). The baseline characteristics of the study population are shown in Table 1. Among the patients included in the study cohort, all patients had a diagnosis of inflammatory bowel disease based on ICD codes: 5059 (54.1\%) had a diagnosis of CD [555.* (ICD-9) and K50* (ICD-10)], 4648 (49.7\%) had a diagnosis of UC [556** (ICD-9) and K51.* (ICD-10)], 135 (1.4\%) had indeterminate colitis [558.9 (ICD-9), K52.3 (ICD-10)], and 47 (0.5\%) had pouchitis [569.7* (ICD-9) and K91.85* (ICD-10)]. There were 505 patients had overlapping ICD codes. We identified 12,595 samples from the 9356 patients who were included in the study. Longitudinal samples were available for $2161(23.1 \%)$ patients, of whom $70.6 \%(1526 / 2161)$ had 2 samples. The median number of tests carried out per patient was 1 (range: 1-25) with $99.9 \%$ of the patients with a range of 1-7 tests. 


\section{Vedolizumab Levels and Anti-vedolizumab Antibodies}

The median VDZ serum level in the per sample analysis was $12 \mu \mathrm{g} / \mathrm{mL}$ (IQR: $6.4-22.1 \mu \mathrm{g} / \mathrm{mL}$ ). Thirty-seven percent of patients (3470) had VDZ levels $\geq 15 \mu \mathrm{g} / \mathrm{mL}$ at presentation when the first specimen was analyzed. VDZ levels were significantly higher among the pediatric study population ( $<18$ years) when compared to adults [3.6 $\mu \mathrm{g} / \mathrm{mL}(6.4-25.6)$ vs. $11 \mu \mathrm{g} / \mathrm{mL}(5.6-20), p<0.0001]$.

Of the entire patient population, 274 patients had detectable ATV (2.9\%) in one or more of the available tests per patient, while the percentage of per sample detectable ATV was $2.4 \%$ (303 of 12,595 samples). A total of 201 samples from 184 patients (2\%) had both detectable VDZ levels and ATV. The rate of detectable ATV was not significantly higher in the adult versus the pediatric population [OR 1.1 (95\% CI 0.62-1.75), $p=0.7]$.

We performed a per-patient analysis using the first available sample for those patients who never presented with detectable ATV $(n=9082)$ and compared it to those patients using the first ATV-positive sample where ATV was detected in at least one sample $(n=274)$. The median VDZ concentration in patients who never developed ATV was significantly higher versus the first ATV-positive sample drawn on patients who did develop positive ATVs [11.3 $\mu \mathrm{g} /$ $\mathrm{mL}(5.9-20.6)$ vs. $3.9 \mu \mathrm{g} / \mathrm{mL}(0-9.0, p<0.0001]$ (Fig. 1). Likewise, in a per-sample analysis, the median VDZ concentration was also significantly higher in those samples with undetectable ATV when compared to those with detectable ATV [12.2 $\mu \mathrm{g} / \mathrm{mL}$ (6.6-22.3) vs. $4.3 \mu \mathrm{g} / \mathrm{mL}$ (0-9.6)] (Supplementary Figure 1A). ATV were detectable at all drug

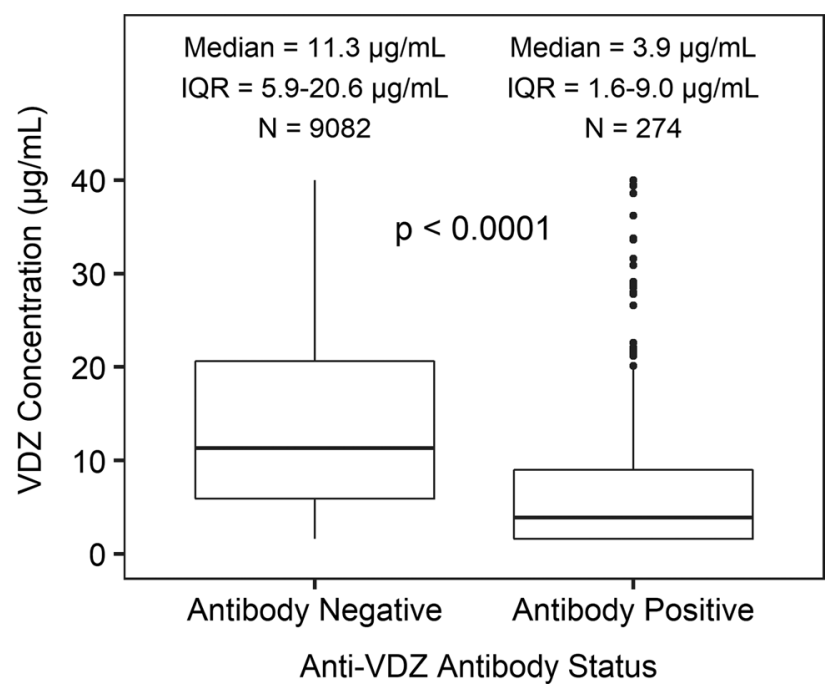

Fig. 1 Vedolizumab concentrations in the first available sample in patients with and without detectable anti-vedolizumab antibodies. Median (IQR) vedolizumab concentrations are shown concentrations; however, the percentages of antibody positive samples were the highest at the lowest drug concentrations (Supplementary Figure 1B). A total of $95 \%$ of ATVpositive samples were detectable at or below the VDZ drug concentration of $31.5 \mu \mathrm{g} / \mathrm{mL}$ (data not shown).

When performing sub-group analysis in patients with $\mathrm{CD}$ and UC, we found similar results. Patients with CD who were ATV-positive had significantly lower median VDZ levels versus those without ATV [4.1 $\mu \mathrm{g} / \mathrm{mL}$ (IQR: 0.0-9.3) vs. $11.1 \mu \mathrm{g} / \mathrm{mL}$ (IQR: 5.6-20.4), $p<0.0001]$. Furthermore, those patients with CD and detectable ATV had a lower chance of achieving VDZ levels $\geq 15 \mu \mathrm{g} / \mathrm{mL}$ [OR 0.33 (95\% CI $0.17-0.58), p<0.0001]$. In the UC sub-group, we also found a significant difference in drug levels between those patients with and without ATV [3.2 $\mu \mathrm{g} / \mathrm{mL}$ (IQR: 0.0-7.6) vs. $11.5 \mu \mathrm{g} / \mathrm{mL}$ (IQR: $6.1-20.7$ ), $p<0.0001$ ]. As in the CD sub-group, those patients with UC and detectable ATV had a lower chance of achieving a VDZ level $\geq 15 \mu \mathrm{g} / \mathrm{mL}$ [OR 0.23 (95\% CI 0.13-0.37), $p<0.0001]$.

\section{Endoscopic Healing Index, Vedolizumab Serum Levels, and Anti-vedolizumab Antibodies}

A total of 287 patients had VDZ/ATV levels and EHI performed within 30 days of each other. Of those, 59\% were women and the median age was 48 years (IQR: 36-62) (Table 1). $3.8 \%$ were pediatric patients. Ninety-one percent of the matching VDZ and EHI tests were performed on the same serum sample and $95 \%$ were performed on samples collected within one day of each other. The median EHI of the cohort was 36 (IQR: 23-51) and the median VDZ levels were $14.1 \mu \mathrm{g} / \mathrm{mL}$ (IQR: 7.1-24.7). Only 4 of the 287 patients had detectable ATV (1.4\%). As in the total cohort of the study patients (those with and without a EHI test performed), VDZ levels were significantly lower in those patients with detectable ATV when compared to those without ATV $(<1.6 \mu \mathrm{g} / \mathrm{mL}[\mathrm{IQR}:<1.6-2.18]$ vs. $14.5 \mu \mathrm{g} / \mathrm{mL}$ [IQR: 7.4-25.0], $p=0.003)$.

When stratifying the patients into 3 groups based on their EHI values using the two established thresholds (EHI of 20 and 50), serum VDZ levels were significantly higher in those patients with $\mathrm{EHI}<20$ when compared to those with EHI between 20 and 50 and those with EHI $>50(p=0.006$ and 0.0014 respectively-Fig. 2a). A negative regression of EHI values (slope: $-3.304, p=0.0016$ ) was observed with increasing VDZ drug quartile values (supplementary Figure 2 and supplementary Table 1) with increasing likelihood of patients with EHI $<20$ (Fig. 2b, Table 2). Patients with a VDZ level $\geq 15 \mu \mathrm{g} / \mathrm{mL}(n=134)$ were significantly more likely to have an EHI < 20 (OR 3.5 [95\% CI 1.8-7.3], $p<0.0001)$ compared to patients with VDZ concentrations $<15 \mu \mathrm{g} / \mathrm{mL}(n=153)$. 

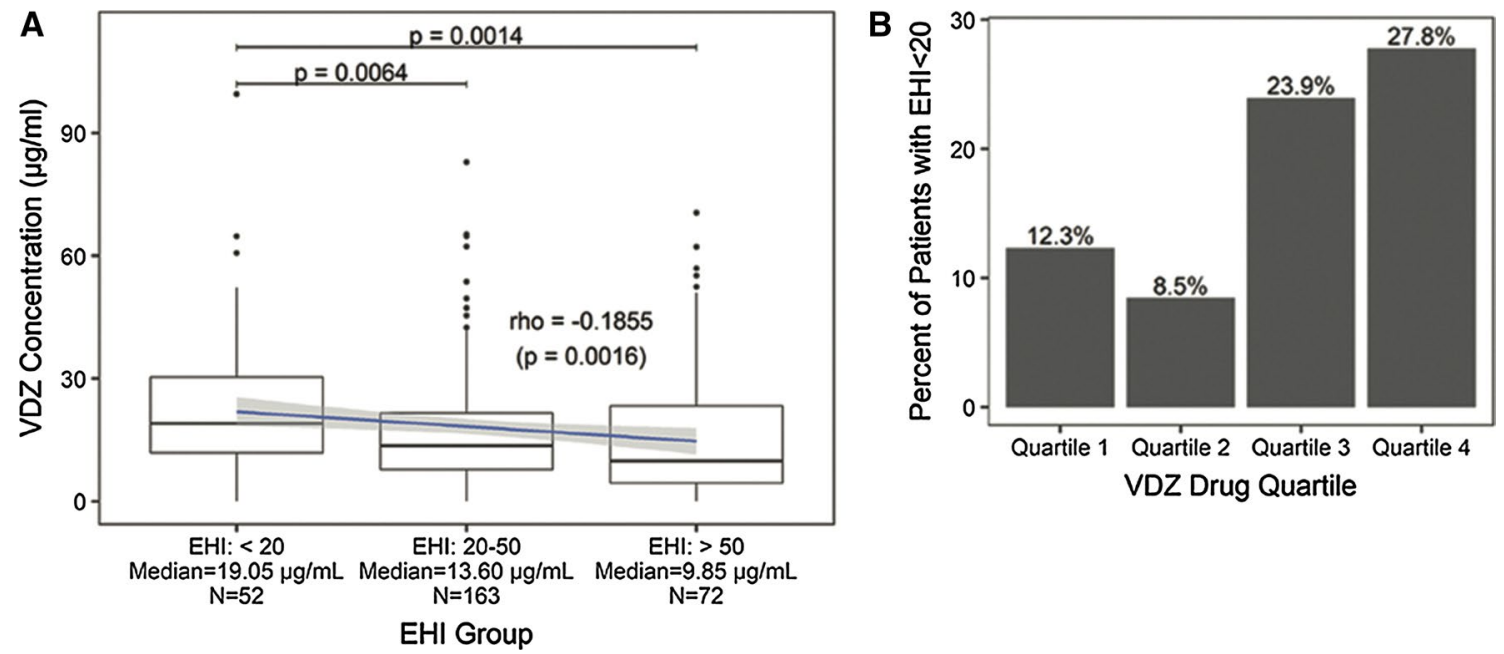

Fig. 2 a)Serum drug concentrations of vedolizumab in endoscopic healing index (EHI) groups of $<20$, between 20 and 50 and $>50$ are presented with median values indicated below each EHI group. Spearman rho value indicating linear inverse relation between vedoli-

Table 2 Vedolizumab concentration ranges in each quartile and corresponding percentage of patients with Endoscopic healing Index $<20$ in each drug quartile

\begin{tabular}{lllc}
\hline $\begin{array}{l}\text { VDZ drug } \\
\text { quartile }\end{array}$ & $\begin{array}{l}\text { VDZ concentration } \\
\text { range }(\mu \mathrm{g} / \mathrm{mL})\end{array}$ & $N$ & $\mathrm{EHI}<20 N(\%)$ \\
\hline 1 & $0.0-7.1$ & 73 & $9(12.3 \%)$ \\
2 & $7.2-14.1$ & 71 & $6(8.5 \%)$ \\
3 & $14.2-24.7$ & 71 & $17(23.9 \%)$ \\
4 & $\geq 24.8$ & 72 & $20(27.8 \%)$ \\
\hline
\end{tabular}

$V D Z$ Vedolizumab, EHI endoscopic healing index

\section{Endoscopic Healing Index Threshold Analysis}

The VDZ drug threshold that differentiates an EHI $<20$ and an EHI $>50$ with the best sensitivity/specificity (Youden's index) was identified as $15.7 \mu \mathrm{g} / \mathrm{ml}$ sensitivity $(95 \%$ zumab concentrations and increasing EHI groups is presented. b Proportion of Crohn's disease patients with an endoscopic healing index $(\mathrm{EHI})<20$ by serum concentration quartile of vedolizumab

$\mathrm{CI})=65.3 \%(53.1-76.1)$ and specificity $(95 \% \mathrm{CI})=71.2 \%$ (56.9-82.9) (Fig. 3a).

When analyzing the study cohort using a VDZ drug threshold of $15.7 \mu \mathrm{g} / \mathrm{mL}, 72(25.1 \%)$ patients treated with VDZ were identified as having an EHI $>50$ of whom 47 (65.2\%) had a VDZ concentration $<15.7 \mu \mathrm{g} / \mathrm{mL}$ (Fig. 3b). Conversely, $35 \%$ of patients on VDZ were identified to have EHI $>50$ with VDZ concentration $\geq 15.7 \mu \mathrm{g} / \mathrm{ml}$.

\section{Conclusion}

A large amount of evidence has supported the use of TDM as a tool to manage patients with IBD receiving biologics, especially anti-TNF agents. The role of TDM in patients being treated with VDZ has been less recognized. There may be several reasons that can explain this phenomenon.
Fig. 3 a ROC curve of vedolizumab drug concentrations for differentiating patients with EHI $>50$ versus $\mathrm{EHI}<20$. b Vedolizumab contingency tables with sensitivity and specificity at a serum vedolizumab concentration threshold at the Youden's index $(15.7 \mu \mathrm{g} / \mathrm{mL})$

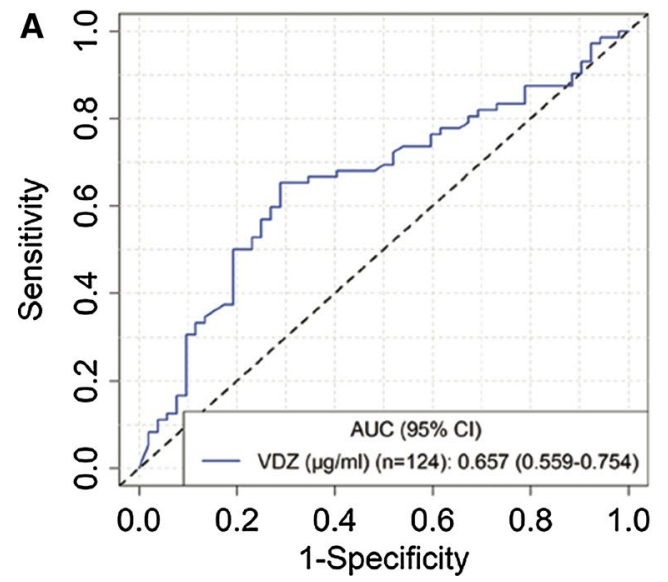

B

\begin{tabular}{|c|c|c|c|}
\hline & $\mathrm{EHI}>50$ & $\mathrm{EHI}<20$ & Sum \\
\hline Drug<15.7 & $47(\mathrm{TP})$ & $15(\mathrm{FP})$ & 62 \\
\hline Drug $\geq 15.7$ & $25(\mathrm{FN})$ & $37(\mathrm{TN})$ & 62 \\
\hline Sum & 72 & 52 & 124 \\
\hline \multicolumn{3}{|c|}{ Sensitivity (95\%Cl): $\mathbf{6 5 . 3 \%}(\mathbf{5 3 . 1}-\mathbf{7 6 . 1 )}$} \\
Specificity (95\%Cl): $\mathbf{7 1 . 2 \%}(\mathbf{5 6 . 9} \mathbf{8 2 . 9 )}$
\end{tabular}


Most previous studies have found a low rate of immunogenicity against VDZ. This has restricted analyses exploring the relationship of ATV and the pharmacokinetics of VDZ. There is also a gap in knowledge on how TDM can be used in patients on VDZ, especially when using treat-to-target strategies and biomarkers.

In this study, we analyzed serum VDZ and ATV concentrations from almost 10,000 adult and pediatric patients treated with VDZ. This allowed us to understand the association of VDZ levels and the presence of ATV. We found that the presence of ATV was associated with lower VDZ drug levels, suggesting that those patients who do develop immunogenicity to VDZ can theoretically be at a higher risk of experiencing non-response or an infusion reaction to the drug [14]. Despite the heterogeneity of assays used, the rates of immunogenicity in other cohorts have been similar [1,2]. In a study that also used the HMSA to assess the correlation between VDZ levels, ATV, and remission in patients receiving maintenance therapy for $\mathrm{UC}$ or $\mathrm{CD}$, the authors found that $1.6 \%$ of the study population had ATV [13]. In another study using a drug-tolerant assay, only 1 of 928 samples had detectable ATV. Rates of immunogenicity in the pivotal GEMINI I and II trials were 3.7 and $4.1 \%$, respectively [1, 2].

Future studies looking to identify patients with transient versus persistent antibodies and their clinical significance are warranted. A prospective trial looking into VDZ levels during induction and long-term outcomes found that, of 55 patients, 3 had detectable antibodies at week 2 , and they all became undetectable by week 6 [8].

This study also explored the association between serum VDZ levels and the EHI, a non-invasive serum biomarker with favorable diagnostic accuracy for identifying mucosal inflammation in patients with CD. We found that VDZ levels were significantly higher in those patients with an EHI $<20$ (a threshold strongly associated with endoscopic healing), suggesting that VDZ levels may correlate with a lack of endoscopic mucosal inflammation. In a threshold analysis, we found that a VDZ level $>15.7 \mu \mathrm{g} / \mathrm{ml}$ was best associated with an EHI $<20$. Further studies looking into the association between the EHI, drug levels and response to VDZ are warranted. Furthermore, it is important to consider that there are several assays available to measure VDZ levels and ATV in serum. This is critical, as the specific results of this study may not necessarily be the same if another assay is used. [13]. As pointed out in previous studies, an important question is how to explain these findings considering that previous studies have found that target membrane $\alpha 4 \beta 7$ integrin on peripheral blood memory $\mathrm{T}$ cells are fully occupied during both induction and maintenance with VDZ, regardless of clinical response or drug serum levels [6]. Despite the lack of knowledge whether or not all samples were collected at trough, these data suggest an apparent exposure-response relationship in VDZ, and that VDZ exposure concentrations $>15.7 \mu \mathrm{g} /$ $\mathrm{ml}$ are associated with $\mathrm{EHI}<20$.

The study had the benefit of analyzing real-world data from a large adult and pediatric population receiving VDZ. To the best of our knowledge, this is the largest study reporting rates of immunogenicity against VDZ, allowing the exploration of how ATVs affect drug levels (and, hence, potentially efficacy). Previous studies have been limited due to the relatively low number of subjects presenting with detectable ATV, significantly limiting the analysis. Our large study population also allowed for sub-group analysis. For example, we found no difference in rates of immunogenicity between adults and pediatric populations or between CD and UC patients.

This study is also the first to report the association between VDZ levels, ATV, and EHI in patients with CD. EHI is a novel algorithm which includes 13 biomarkers that has been shown to have a good correlation with endoscopic mucosal inflammation, hence being a potentially good non-invasive tool to be used in treat-to-target strategies. The study did have several limitations, including the cross-sectional design, lack of clinical data such as disease phenotype and severity, drug frequency, timing of the blood draw within the dose interval, the lack of knowledge on why the test was performed (pro-active monitoring, primary non-response, secondary non-response, infusion reaction, or re-start after a drug holiday), and the action taken by the treating clinician to the test results (such as dose escalation and adding an immunomodulator).

In conclusion, although rates of ATV are low and transient in most patients, they are significantly associated with lower serum VDZ levels. Furthermore, serum VDZ levels also demonstrated a significantly inverse association with EHI. The use of TDM and treat-to-target strategies in patients on VDZ warrant further investigation.

\section{Compliance with Ethical Standards}

Disclosures Andres J Yarur: Consultant/Advisory boards Takeda and Prometheus Biosciences. Speaker fees for Takeda and Prometheus Biosciences. Parakkal Deepak: supported by a Junior Faculty Development Award from the American College of Gastroenterology. Consultant or advisory board member for Janssen, Pfizer and Prometheus, speaker's bureau for AbbVie; research grant from Takeda. Niels Vande Casteele: research grants from R-Biopharm; grants and personal fees from Takeda and UCB; and personal fees from Alimentiv, Inc. (formerly Robarts Clinical Trials, Inc.), Celltrion and Prometheus. Robert Battat: no conflict of interests. Anjali Jain: Employee of and owns stock options for Prometheus Biosciences. Lauren Okada: Employee of Prometheus Biosciences. Mark Osterman: advisory board consultant for AbbVie, Elan, Genentech/Roche, Janssen, Lycera, Merck, Pfizer, Takeda, UCB; research grant support from UCB. Miguel Regueiro; Advisory Boards and Consultant for Abbvie, Janssen, UCB, Takeda, Pfizer, Miraca Labs, Amgen, Celgene, Seres, Allergan, Genen- 
tech, Gilead, Salix, Prometheus, Lilly, TARGET Pharma Solutions, ALFASIGMA, S.p.A.

Ethical approval De-identified information was extracted from the clinical laboratory database. Clinical information that did not include the Health Insurance Portability and Accountability Act (HIPAA) identifiers were used for these analyses. The data were extracted from the Prometheus Biosciences database, National Institutes of Health criteria for exemption from human subject's regulations (category 4) were met as all personal information was removed to protect privacy. This included all subject data being de-identified and the removal of all HIPAA identifiers prior to data extraction. The dataset was maintained with no possibility of identification of personal information. Data extraction was reviewed and approved by the Prometheus Biosciences privacy office for compliance. All authors had access to study data, and reviewed and approved the final manuscript.

Open Access This article is licensed under a Creative Commons Attribution-NonCommercial 4.0 International License, which permits any non-commercial use, sharing, adaptation, distribution and reproduction in any medium or format, as long as you give appropriate credit to the original author(s) and the source, provide a link to the Creative Commons licence, and indicate if changes were made. The images or other third party material in this article are included in the article's Creative Commons licence, unless indicated otherwise in a credit line to the material. If material is not included in the article's Creative Commons licence and your intended use is not permitted by statutory regulation or exceeds the permitted use, you will need to obtain permission directly from the copyright holder. To view a copy of this licence, visit http://creativecommons.org/licenses/by-nc/4.0/.

\section{References}

1. Feagan BG, Rutgeerts P, Sands BE, et al. Vedolizumab as induction and maintenance therapy for ulcerative colitis. $N$ Engl J Med. 2013;22:699-710.

2. Sandborn WJ, Feagan BG, Rutgeerts P, et al. Vedolizumab as induction and maintenance therapy for crohn's disease. $N$ Engl $J$ Med. 2013;22:711-721.

3. Afif W, Loftus EV, Faubion WA, et al. Clinical utility of measuring infliximab and human anti-chimeric antibody concentrations in patients with inflammatory bowel disease. Am J Gastroenterol. 2010;9:1133-1139.
4. Yarur AJ, Jain A, Hauenstein SI, et al. Higher Adalimumab levels are associated with histologic and endoscopic remission in patients with Crohn's disease and ulcerative colitis. Inflamm Bowel Dis. 2016;22:409-415.

5. Ungar B, Levy I, Yavne Y, et al. Optimizing anti-TNF- $\alpha$ therapy: serum levels of infliximab and adalimumab are associated with mucosal healing in patients with inflammatory bowel diseases. Clin Gastroenterol Hepatol. 2016;14:e2.

6. Ungar B, Kopylov U, Yavzori M, et al. Association of vedolizumab level, anti-drug antibodies, and $\alpha 4 \beta 7$ occupancy with response in patients with inflammatory bowel diseases. Clin Gastroenterol Hepatol. 2017;16:1-34.

7. Osterman MT, Rosario M, Lasch K, et al. Vedolizumab exposure levels and clinical outcomes in ulcerative colitis: determining the potential for dose optimisation. Aliment Pharmacol Ther. 2019;20:408-418.

8. Yarur AJ, Bruss A, Naik S, et al. Vedolizumab concentrations are associated with long-term endoscopic remission in patients with inflammatory bowel diseases. Dig Dis Sci. 2019;5:1651-1659. https://doi.org/10.1007/s10620-019-05570-1.

9. Colombel J-F, Panaccione R, Bossuyt P, et al. Effect of tight control management on Crohn's disease (CALM): a multicentre, randomised, controlled phase 3 trial. Lancet. 2018;23:2779-2789.

10. Baert F, Moortgat L, Van Assche G, et al. Mucosal healing predicts sustained clinical remission in patients with early-stage Crohn's disease. Gastroenterology. 2010;138:463-468.

11. D'Haens G, Kelly O, Battat R, et al. Development and validation of a test to monitor endoscopic activity in patients with Crohn's disease based on serum levels of proteins. Gastroenterology. 2020;158:e10.

12. Wang S-L, Ohrmund L, Hauenstein S, et al. Development and validation of a homogeneous mobility shift assay for the measurement of infliximab and antibodies-to-infliximab levels in patient serum. J Immunol Methods. 2012;382:177-188.

13. Ungaro RC, Yarur A, Jossen J, et al. Higher trough vedolizumab concentrations during maintenance therapy are associated with corticosteroid-free remission in inflammatory bowel disease. $J$ Crohn's Colitis. 2019;13:1741-1747.

14. Feagan BG, Greenberg GR, Wild G, et al. Treatment of ulcerative colitis with a humanized antibody to the alpha4beta7 integrin. $N$ Engl J Med. 2005;16:2499-2507.

Publisher's Note Springer Nature remains neutral with regard to jurisdictional claims in published maps and institutional affiliations. 